\title{
A Method for the Identification of Inaccuracies in Pupil Segmentation
}

\author{
Hugo Proença and Luís A. Alexandre \\ Dep. Informatics, IT - Networks and Multimedia Group \\ Universidade da Beira Interior, Covilhã, Portugal \\ Email: \{hugomcp,lfbaa\}@di.ubi.pt
}

\begin{abstract}
In this paper we analyze the relationship between the accuracy of the segmentation algorithm and the error rates of typical iris recognition systems. We selected 1000 images from the UBIRIS database that the segmentation algorithm can accurately segment and artificially introduced segmentation inaccuracies. We repeated the recognition tests and concluded about the strong relationship between the errors in the pupil segmentation and the overall false reject rate. Based on this fact, we propose a method to identify these inaccuracies.
\end{abstract}

Keywords: biometrics, image extraction, image segmentation, iris recognition.

\section{Introduction}

The use of biometric systems has been increasingly encouraged by both government and private entities in order to replace or improve traditional security systems. Iris is commonly recognized as one of the most reliable biometric measures: it has a random morphogenesis and no genetic penetrance.

An iris recognition system can be seen as an image processing problem and, as in any other image processing task, the segmentation plays an important role in the final results. Moreover, in spite of the accuracy of the segmentation algorithms, the dynamic conditions of the environment where systems are functioning can easily contribute for the existence of segmentation inaccuracies.

Our aim consists in the analysis of the relationship between the accuracy of the segmentation process and the error rates of the recognition system. In order to achieve this objective, we implemented the following process:

1. Selection of 1000 images from the UBIRIS [12] database. Verification that the used segmentation algorithm can accurately segment all the images.

2. Feature extraction 1: extraction of the iris signatures following three distinct feature extraction methodologies that represent the most common iris recognition approaches.

3. Feature comparison 1: comparison of the resultant iris signatures using the Hamming distance.

4. Introduction of segmentation inaccuracies. Corruption of the segmentation algorithm in order to identify the iris borders in a less accurate way.

5. Feature extraction + comparison 2: extraction and comparison of the resulting signatures through the previously used methods.

This analysis is obviously independent of the choice of segmentation algorithm, as we manually verified that the used one was able to accurately identify both the iris borders (pupillary and scleric) from all images of the data set. On the other hand, it is dependent of the three tested feature extraction methodologies ([4], [1] and [9]), that have as common points the utilization of normalized and dimensionless iris images [4] and the creation of binary iris signatures.

The remaining sections of this paper are organized as follows: in section 2 we present the main stages of the classical iris recognition systems and an overview of the most cited iris segmentation methodologies. Section 3 analyzes the contribution of each type of segmentation error for the accuracy of the recognition system. On section 4 we describe the proposed methodology and present its results on section 5. Finally, section 6 lists the conclusions.

\section{Iris Recognition Systems}

In spite of the heterogeneity of the approaches proposed by different authors, typical iris recognition systems share a common structure that is given in figure 1 . 


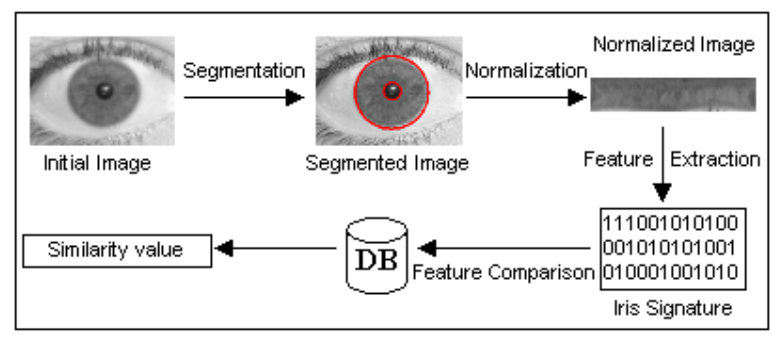

Figure 1. Typical stages of the iris recognition systems

The initial stage deals with iris segmentation. This process consists in, approximating the iris as a circumference or an ellipse, localize the iris inner (pupillary) and outer (scleric) borders. Some authors also propose methods for the detection of eyelids and eyelashes regions that may obstruct portions of the iris.

In order to compensate the varying size of the pupil it is common to translate the segmented iris image represented in the cartesian coordinate system to a fixed length and dimensionless image in the polar coordinate system.

The next stage is the feature extraction. From this viewpoint, iris recognition approaches can be divided in three major categories: phase-based methods (e.g. [4]), zero crossing methods (e.g. [2] and [10]) and texture analysis based methods (e.g [14], [7] and [8]). All these methods produce a set of numeric values known as an biometric iris signature.

The final stage consists on the comparison of irises signatures, producing each one a numeric dissimilarity value. If this value is superior to a threshold, the system outputs a "non-match", meaning that each signature belongs to different subjects. Otherwise, the system outputs a "match", meaning that both signatures belong to the same person. In this process, different distance metrics are applied (e.g. Hamming, Euclidean, Weighted Euclidean, ... ) or methods based on signal correlation.

As figure 1 illustrates, the segmentation act as the basis for the following stages. In the next sub-section we briefly describe the most common iris image segmentation methodologies.

\subsection{Iris Segmentation Methodologies}

There are currently two major strategies for iris segmentation: using a rigid or deformable template of the iris or its boundary. In most cases, the boundary approach begins by the construction of an edge map followed by the application of some geometric form fitting algorithm. The templatebased strategies usually involves the maximization of some equation and are in general more specific.
Since 1987, when the first relevant methodology was presented by Flom and Safir [5], many distinct approaches have been proposed. In 1993, J. Daugman [4] presents one of the most cited methodologies, constituting the basis of many functioning systems. On the segmentation stage, this author introduces an integrodifferential operator to find both the iris inner and outer borders. This operator remains up to date and was proposed with some minor differences in 2004 by [11].

In a similar form, [3] and [10] propose integrodifferential operators that search over a $N^{3}$ space having as objective the maximization of equations that identify the iris borders.

Wildes [14] proposes iris segmentation through a gradient based binary edge map construction followed by the application of the circular Hough transform. This is the most common methodology, being proposed with minor variants by [6], [8], and [9]. In [13], the authors propose a methodology also based in Wildes' method but use a clustering process to achieve robustness for non-cooperative environments.

Based on the assumption that the image captured intensity values can be well represented by a mixture of three Gaussian distribution components, authors of [7] propose the use of the Expectation Maximization algorithm to estimate the respective distributions parameters.

\section{Influence of the Segmentation Errors in the Recognition's Accuracy}

In this section we describe the tested segmentation errors as well analyze their influence on the results of the recognition system.

In the segmentation stage we implemented the method proposed by Wildes [14]. We manually verified that the algorithm accurately identified the pupillary and scleric iris borders of all images of our data set (section 3.1).

The cartesian to polar transformation was made through the widely used Daugman Rubber Sheet [4], producing as output a dimensionless polar representation of the iris with fixed dimensions of 512 (width) by 64 (height) pixels.

Three distinct feature extraction methodologies were implemented. These methodologies are described respectively in [4], [1] and [9] and share the fact that they all produce binary iris signatures. This enabled the comparison through the Hamming distance, as described in [4].

\subsection{Experiments Database}

All images used were extracted from the UBIRIS [12] database, known by its highly heterogeneous image characteristics, regarding focus, reflections, brightness and contrast parameters. 
We selected 10 images from 100 subjects, giving a total of 1000 images. At each iteration of the test, every image was processed and the resulting signature compared with all remaining ones, enabling 4500 intra-class and 495000 inter-class comparisons (respectively comparisons between images from the same and different subjects).

Images have fixed dimensions of 400 (width) by 300 (height) pixels and horizontal and vertical resolution of 300 dpi. The irises have radius values between 80 and 100 pixels and the pupils radius values between 15 and 35 pixels.

\subsection{Types of Segmentation Errors}

The used iris segmentation methodology [14] approximates both the scleric and pupillary borders as circumferences, thus each of the borders can be defined by its center coordinates $(x, y)$ and radius $r$. Let $\left(x_{d}, y_{d}\right)$ and $r_{d}$ be respectively the center coordinates and radius of the detected circumference. Also let $\left(x_{t}, y_{t}\right)$ and $r_{t}$ be the circumference parameters of the true iris border.

On the implementation of the segmentation algorithm we artificially introduced two types of error:

- Translation Errors: we defined a translation error of $p$ pixels when $\left\|x_{d}-x_{t}\right\|+\left\|y_{d}-y_{t}\right\|=p$. It occurs when the center of the detected circumference is deviated $p$ pixels from the center of the true circumference. Figure 2a shows an example of a translation error in the pupillary border.

- Scale Errors: as figure 2b illustrates, a scale error occurs when the detected and the true circumference have different radius values. If $\left\|r_{d}-r_{t}\right\|=p$ then we considered it as a scale error of $p$ pixels.

These two types of errors on each iris border enabled the appearance of four distinct segmentation errors: translation error on the scleric border, translation error on the pupillary border, scale error on the scleric border and scale error on the pupillary border.

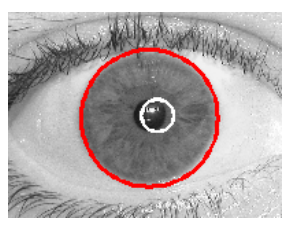

(a) Translation error on the segmented pupillary border

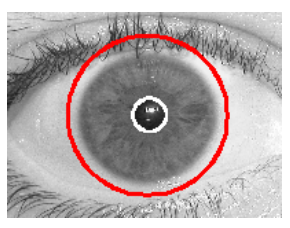

(b) Scale error on the segmented scelric border
Figure 2. Inaccurately segmented iris images.

\subsection{Results and Discussion}

By using Hamming distance as the similarity measure, each comparison between irises signatures produces a dissimilarity value in the $[0,1]$ interval, directly proportional to the compared irises dissimilarity.

At each iteration of our test, we made all the possible intra-class and inter-class iris comparisons with different types and values of segmentation errors.

Table 1 contains the average values resultant from the image comparisons using each of the above mentioned feature extraction methodologies. The first column indicates the segmentation error, the second, third, forth and fifth contain respectively the average and the standard deviation from the values obtained in the comparisons between the same (intra-class) and different (inter-class) irises. The last four columns are related with the obtained error rates. At each test we adjusted the similarity threshold (sixth column) in order to minimize the false accept rate (seventh column). For the obtained threshold value, we calculated the correspondent false reject rate (eighth column) and the overall error rate (ninth column).

Not surprisingly, we observed that the difference between the average values for the intra-class and inter-class comparisons has decreased when we introduced the segmentation errors.

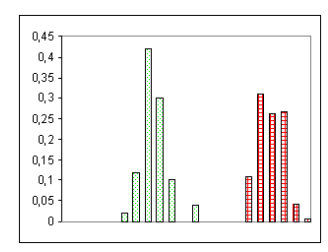

(a) Distance histogram with accurate segmentation

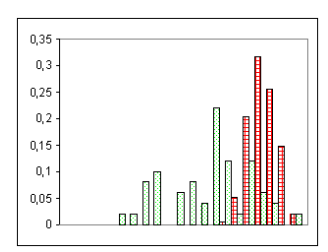

(b) Distance histogram with translation errors of 3 pixels in the pupillary border segmentation

\section{Figure 3. Degradation of the recognition sys- tem's results.}

Moreover, we concluded about the major importance of the accurate segmentation of the pupillary border. In this case, the existence of minor translation errors - just above 1 pixel - significantly increased the false reject rate and deteriorated the overall accuracy of the recognition system.

Figure 3 illustrates the degradation of the results when we introduced translation errors on the pupil segmentation. The first histogram (figure 3a) corresponds to the values obtained without segmentation errors and the second one with translation errors of 3 pixels in the pupil segmentation. The clear and darker bars correspond respectively to the values of intra and inter-class comparisons. 
Table 1. Iris recognition results.

\begin{tabular}{|c|c|c|c|c|c|c|c|c|}
\hline Segmentation Error & Avg. Intra-Class & Std. Intra-Class & Avg. Inter-Class & Std. Inter-Class & Threshold & Min. FAR $(\%)$ & FRR (\%) & Error $(\%)$ \\
\hline No error & 0.1808 & 0.00053 & 0.3926 & 0.00058 & 0.2636 & 0 & 0 & 0 \\
\hline \multicolumn{9}{|c|}{ Translation error on pupillary border } \\
\hline 1 pixel & 0.2332 & 0.00232 & 0.3966 & 0.00049 & 0.3330 & 0 & 0 & 0 \\
\hline 2 pixels & 0.2848 & 0.00494 & 0.4037 & 0.00056 & 0.3388 & 0 & 22 & 3.66 \\
\hline 3 pixels & 0.3185 & 0.00749 & 0.4130 & 0.00078 & 0.3417 & 0 & 40 & 6.66 \\
\hline 4 pixels & 0.3503 & 0.00563 & 0.4178 & 0.00077 & 0.3476 & 0 & 56 & 9.33 \\
\hline 5 pixels & 0.3728 & 0.00406 & 0.4232 & 0.00112 & 0.3212 & 0 & 80 & 13.33 \\
\hline \multicolumn{9}{|c|}{ Translation error on scleric border } \\
\hline 1 pixel & 0.1898 & 0.00079 & 0.3925 & 0.00059 & 0.2763 & 0 & 0 & 0 \\
\hline 2 pixels & 0.2028 & 0.00105 & 0.3977 & 0.00062 & 0.2939 & 0 & 0 & 0 \\
\hline 3 pixels & 0.2083 & 0.00101 & 0.3978 & 0.00059 & 0.2871 & 0 & 0 & 0 \\
\hline 4 pixels & 0.2085 & 0.00115 & 0.3978 & 0.00056 & 0.2988 & 0 & 0 & 0 \\
\hline 5 pixels & 0.2216 & 0.00190 & 0.4013 & 0.00065 & 0.3154 & 0 & 0 & 0 \\
\hline 10 pixels & 0.2561 & 0.00249 & 0.4010 & 0.00072 & 0.3154 & 0 & 10 & 1.66 \\
\hline \multicolumn{9}{|c|}{ Scale error on pupillary border } \\
\hline 1 pixel & 0.1830 & 0.00075 & 0.3925 & 0.00058 & 0.2734 & 0 & 0 & 0 \\
\hline 2 pixels & 0.2000 & 0.00125 & 0.3946 & 0.00061 & 0.3144 & 0 & 0 & 0 \\
\hline 3 pixels & 0.2111 & 0.00175 & 0.4020 & 0.00081 & 0.3037 & 0 & 0 & 0 \\
\hline 4 pixels & 0.2331 & 0.00347 & 0.4040 & 0.00102 & 0.3281 & 0 & 4 & 0.66 \\
\hline 5 pixels & 0.2448 & 0.00449 & 0.4125 & 0.00086 & 0.3095 & 0 & 20 & 3.33 \\
\hline \multicolumn{9}{|c|}{ Scale error on scleric border } \\
\hline 1 pixel & 0.1913 & 0.00075 & 0.3940 & 0.00060 & 0.2773 & 0 & 0 & 0 \\
\hline 2 pixels & 0.2027 & 0.00097 & 0.3981 & 0.00060 & 0.3007 & 0 & 0 & 0 \\
\hline 3 pixels & 0.2143 & 0.00203 & 0.4022 & 0.00074 & 0.3164 & 0 & 0 & 0 \\
\hline 4 pixels & 0.2243 & 0.00178 & 0.4022 & 0.00079 & 0.3173 & 0 & 0 & 0 \\
\hline 5 pixels & 0.2453 & 0.00341 & 0.4121 & 0.00089 & 0.3222 & 0 & 6 & 1 \\
\hline
\end{tabular}

Regarding the iris scleric border, we observed that the recognition systems have a much more tolerant behavior to segmentation errors. This can be explained by two factors: first, the interior part of the iris contains the majority of the information used in the recognition and second, the process that makes the transformation from the cartesian to the polar and dimensionless coordinates system takes the pupil center as basis for its operations.

\section{Proposed Methodology}

If a translation error on the pupil segmentation occurs, some portion of the pupil will be considered as portion of the iris. The pupil is always darker than the iris and we used this fact as the basis for the proposed methodology. Moreover, in the normalized and dimensionless iris image, the wrongly identified portion of the pupil will be located on the upper band of the image (upper left corner from figure 4).

On the upper part of the normalized iris image we computed the average intensity of the pixels located within win-

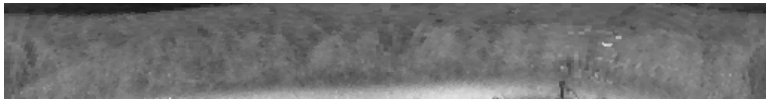

Figure 4. Normalized iris image with a translation error in the pupil segmentation

dows of size $w \times w$ and observed a difference in the distribution of these values. We concluded that on the well segmented irises the values were clearly more homogeneous and experimentally choosed a threshold and window width that can well separate the accurately from the inaccurately segmented pupils.

Formally, let $p_{i, j}$ be the intensity value of the pixel located at row $i$ and column $j$ of the normalized iris image. We computed a set of average values $A=$ $\left\{a_{0}, a_{1}, \ldots, a_{n-1}\right\}$, each $a_{k}$ given by the following equation: 


\section{Table 2. Results of the proposed methodol- ogy.}

\begin{tabular}{|l|c|c|}
\hline Translation Error On Pupillary Border [pixels] & FP $(\%)$ & FN $(\%)$ \\
\hline 0 & 0.38 & 0 \\
\hline 1 & 0 & 2.19 \\
\hline $2,3,4$ and 5 & 0 & 0 \\
\hline
\end{tabular}

$$
a_{k}=\frac{1}{w * w} \sum_{i=0}^{w-1}\left(\sum_{j=k * w}^{(k+1) * w-1} p_{i, j}\right) .
$$

Let $A v g$ be the average value of the elements of $A$ : $A v g=\frac{1}{n} \sum a_{k}$. If exists an $a_{k}$ such that $\left\|a_{k}-A v g\right\|>$ $(T * A v g)$ then the image is classified as containing a translation error in the pupil segmentation.

\section{Method's Results}

In this section we present the results obtained with the proposed method.

Using the same data set and procedure described in section 3, we artificially introduced translation errors in the pupil segmentation and obtained the results contained in table 2. The first column specifies the amplitude of the translation error (number of pixels). The second and third columns contain information respectively about the method's false positives (FP, wrongly classification of "segmentation error") and negatives (FN, wrongly classification of "accurate segmentation").

Our experiments led us to choose the values 0.3 for the threshold parameter $T$ and 3 for the window width $w$. In this case, just about $0.38 \%$ of the bad segmentations reported were wrongly classified. Even on situations where the translation error is minimal (1 pixel), the proposed method has presented low error rates. For translation errors above 1 pixel, the error was equal to zero.

\section{Conclusions}

In this paper we analyzed the important contribution of the accuracy of the segmentation algorithm in the error rates of iris recognition systems. We observed a significant degradation of the recognition rates, specially in the presence of translation errors of the segmented pupil border. In this case, error values higher than 1 pixels significantly increased the false rejection rates.

Based on this fact, we proposed a new method for the identification of translation errors on pupil segmentation.
This method can avoid that the system wrongly outputs a "no match", by redirecting the recognition process to an alternative segmentation process. This fact will obviously increase the comfort of the systems' users as it decreases the false rejection rates.

\section{References}

[1] J. Ali and A. Hassanien. An iris recognition system to enhance e-security environment based on wavelet theory. AMO - Advanced Modeling and Optimization, vol. 6, no. 2, pages 93-104, 2003.

[2] W. W. Boles and B. Boashash. A human identification technique using images of the iris and wavelet transform. IEEE Transactions on Signal Processing, vol. 46, no. 4, pages 1185-1188, April 1998.

[3] T. Camus and R. Wildes. Reliable and fast eye finding in close-up images. In Proceedings of the IEEE 16th International Conference on Pattern Recognition, pages 389-394, 2002.

[4] J. G. Daugman. High confidence visual recognition of persons by a test of statistical independence. IEEE Transactions on Pattern Analysis and Machine Intelligence, vol. 15, no. 11, pages 1148-1161, November 1993.

[5] L. Flom and A. Safir. Iris recognition system, 1987. U.S. Patent 4641394.

[6] J. Huang, Y. Wang, T. Tan, and J. Cui. A new iris segmentation method for recognition. In Proceedings of the 17th International Conference on Pattern Recognition (ICPRO4), vol. 3, pages 23-26, 2004.

[7] J. Kim, S. Cho, and J. Choi. Iris recognition using wavelet features. Kluwer Academic Publishers, Journal of VLSI Signal Processing, no. 38, pages 147-156, November 2004.

[8] L. Ma, Y. Wang, and T. Tan. Iris recognition using circular symmetric filters. In Proceedings of the 15th International Conference on Pattern Recognition (ICPRO2), vol. 2, pages 414-417, 2002.

[9] L. Ma, Y. Wang, and D. Zhang. Efficient iris recognition by characterizing key local variations. IEEE Transactions on Image Processing, vol. 13, no. 6, pages 739-750, June 2004.

[10] D. Martin-Roche, C. Sanchez-Avila, and R.Sanchez-Reillo. Iris recognition for biometric identification using dyadic wavelet transform zero-crossing. IEEE Aerospace and Electronic Systems Magazine, Mag. 17, No. 10, pages 3-6, 2002.

[11] K. Nishino and S. K. Nayar. Eyes for relighting. ACM Trans. Graph., vol 23, no. 3, pages 704-711, 2004.

[12] H. Proença and L. A. Alexandre. UBIRIS: A noisy iris image database. In 13th International Conference on Image Analysis and Processing (ICIAP2005), pages 970-977, September 2005. http: //iris. di . ubi . pt

[13] H. Proença and L. A. Alexandre. Iris segmentation methodology for non-cooperative iris recognition. IEE Proc. Vision, Image \& Signal Processing (To appear), 2006.

[14] R. P. Wildes. Iris recognition: an emerging biometric technology. In Proceedings of the IEEE, vol. 85, no.9, pages 1348-1363, U.S.A., September 1997. 